\title{
Spherical Vortices in Rotating Fluids
}

\author{
By M. M. SCASE \& H. L. TERR Y \\ School of Mathematical Sciences, University of Nottingham, Nottingham NG7 2RD
}

(Received 30 January 2018; revised 9 April 2018; accepted 15 April 2018)

\begin{abstract}
A popular model for a generic fat-cored vortex ring or eddy is Hill's spherical vortex (Phil. Trans. Roy. Soc. A vol. 185, 1894, p. 213). This well-known solution of the Euler equations may be considered a special case of the doubly-infinite family of swirling spherical vortices identified by Moffatt (J. Fluid Mech. vol. 35(1), 1969, p. 117). Here we find exact solutions for such spherical vortices propagating steadily along the axis of a rotating ideal fluid. The boundary of the spherical vortex swirls in such a way as to exactly cancel out the background rotation of the system. The flow external to the spherical vortex exhibits fully nonlinear inertial wave motion. We show that above a critical rotation rate, closed streamlines may form in this outer fluid region and hence carry fluid along with the spherical vortex. As the rotation rate is further increased, further concentric 'sibling' vortex rings are formed.
\end{abstract}

\section{Introduction}

In 1894 Hill published his famous solution for the steady flow of a spherical vortex in an ideal fluid (Hill 1894). The solution consists of an inner rotational spherical region of fluid that matches onto an outer irrotational region of fluid that extends to infinity. Hill's solution was later shown to be the end member of a family of steadily propagating vortex rings (Norbury 1973) of varying core thickness that includes 'thin-cored' rings (see Fraenkel 1970, 1972) where the rotational fluid is confined within a narrow region that does not extend to the axis. These solutions, in particular Hill's, have been the focus of a number of stability analyses (see e.g., Moffatt \& Moore 1978; Pozrikidis 1986; Protas \& Elcrat 2016) that show that in time fluid may be detrained or entrained into the vortex according as to whether it has a prolate or oblate deformation respectively. Hill's spherical vortex may also be viewed as a special non-swirling member of a doubly-infinite family of swirling spherical vortices identified by Moffatt (1969) that may be matched onto an oncoming irrotational stream. Here we show how this family of spherical vortices may be matched onto an oncoming stream in a rotating fluid.

As part of his body of work on rotating fluids in the early twentieth century, Taylor (1922) investigated the response of a rotating fluid to a sphere steadily translating along the axis of rotation. His experiments showed that while the tank of fluid rotated but the sphere was not towed, the sphere rotated with the fluid in solid body rotation. Yet, when the sphere was towed along the axis of rotation it ceased precessing and had no azimuthal velocity in the laboratory frame of reference. Taylor found an exact solution to the Euler equations that supported fully nonlinear inertial waves satisfying a no-slip boundary condition on a sphere that translated steadily along the axis of rotation of the fluid but did not precess about this axis in the laboratory frame (though he noted that it is not clear how such a flow could be realised). In the analysis of this solution Taylor found that in the limit of the radius of the sphere tending to zero, a structure 


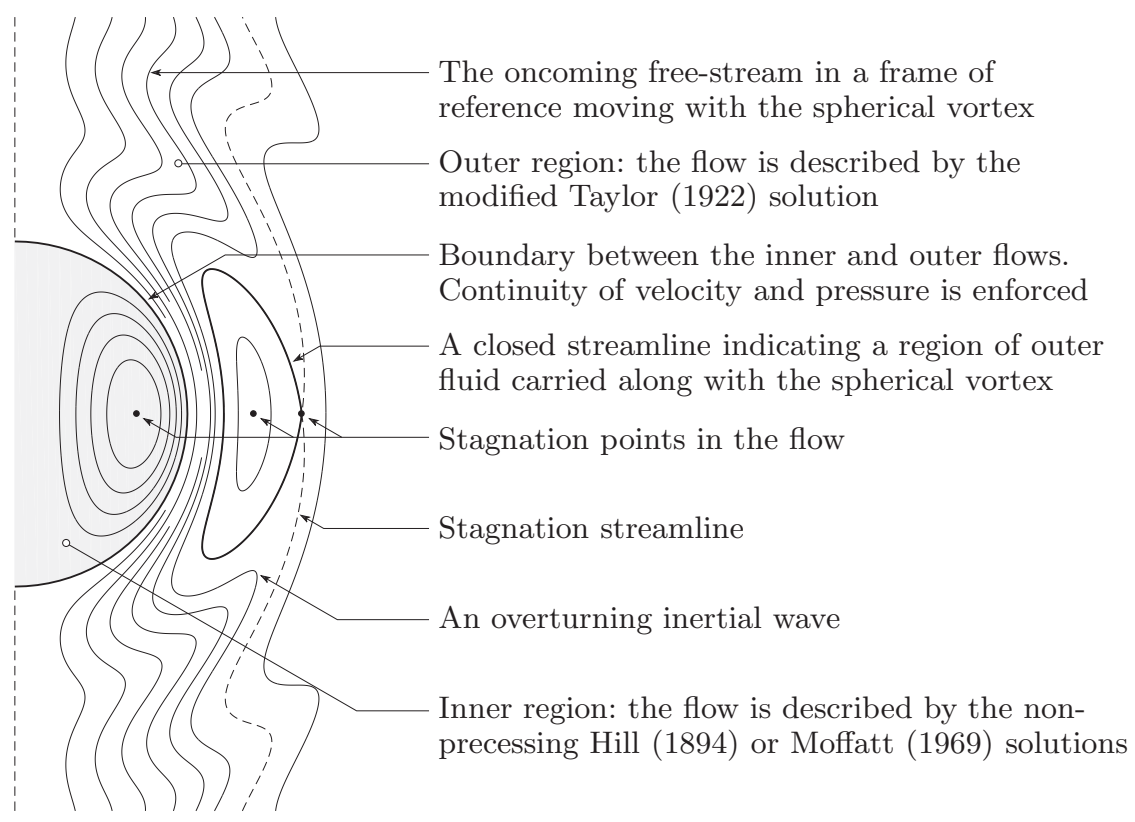

Figure 1: Schematic of the flow in a frame of reference moving with the spherical vortex. The grey inner region consists of either the non-precessing Hill (1894) or Moffatt (1969) solutions. This inner region matches onto a rotating outer region that is given by a modified version of the solution presented by Taylor (1922). At the boundary between the inner and outer regions continuity of velocity and pressure is enforced.

that resembled Hill's spherical vortex could be observed in the flow, though he described this analogy between his flow and Hill's spherical vortex as 'only superficial'.

In the present work we combine the approach of Taylor (1922) (summarized briefly in $\S 2.1$ ) with the solutions of Hill (1894) in $\S 2.2$ and Moffatt (1969) in $\S 2.3$ to find exact solutions of the rotating Euler equations for a spherical vortex propagating steadily along the axis of rotation where, like Taylor's sphere, the boundary of the spherical vortex does not precess in the laboratory frame. The flow, in a frame of reference moving with the spherical vortex, is shown schematically in figure 1 . The inner solution, given by either Hill (1894) or Moffatt (1969), is shown in grey. This inner solution for the spherical vortex is matched onto a modified Taylor (1922) solution in the outer region by enforcing continuity of velocity and pressure across the boundary. The flow in the outer region exhibits inertial waves that above critical rotation rates may overturn. In $\S 2.4$ we show that as the rotation rate of the system is increased above these critical rotation rates, closed streamlines form in the outer fluid representing a series of thin-cored 'sibling' vortex rings, propagating with the spherical vortex. In $\S 3$ we draw our conclusions.

\section{Spherical Vortices in Rotating Fluids}

We consider an inviscid, incompressible fluid with pressure field $p$, velocity field $\boldsymbol{u}$ and constant density $\rho$ in a frame of reference that is rotating with rotation vector $\Omega \hat{\boldsymbol{z}}$, where $\hat{\boldsymbol{z}}$ is a fixed unit vector. The flow is described in terms of spherical polar coordinates $(\sigma, \theta, \phi)$ where $\sigma \geqslant 0$ is the radial distance from the origin, $\theta \in[0, \pi]$ is the polar angle and $\phi \in[0,2 \pi)$ is the azimuthal angle. The spherical coordinate system is aligned such 
that $\theta=0$ is in the $\hat{\boldsymbol{z}}$-direction. We seek to model a spherical vortex of constant radius $a$ and constant propagation velocity $U \hat{\boldsymbol{z}}$, hence we nondimensionalize position as $\boldsymbol{x}=a \tilde{\boldsymbol{x}}$, velocity as $\boldsymbol{u}=U \tilde{\boldsymbol{u}}$, and pressure as $p=\rho U^{2} \tilde{p}$, where tildes indicate nondimensional quantities. Dropping the tildes immediately the nondimensional steady rotating Euler equations that govern the motion are

$$
\nabla \cdot \boldsymbol{u}=0, \quad(\boldsymbol{u} \cdot \nabla) \boldsymbol{u}=-\nabla p+\frac{r}{4 \mathrm{Ro}^{2}} \hat{\boldsymbol{r}}-\frac{1}{\mathrm{Ro}} \hat{\boldsymbol{z}} \times \boldsymbol{u}, \quad \text { Ro }=\frac{U}{2 a \Omega},
$$

where: we have defined a Rossby number, Ro; $\hat{\boldsymbol{r}}=\sin \theta \hat{\boldsymbol{\sigma}}+\cos \theta \hat{\boldsymbol{\theta}}$ is a unit vector in the cylindrical radial direction, and $r=\sigma \sin \theta$ is the cylindrical radial position.

\subsection{Taylor's Solution}

Taylor (1922) considered the axisymmetric flow around a non-precessing sphere (in the laboratory frame) that is translating steadily with nondimensional velocity $\hat{z}$ through a fluid rotating steadily about $\hat{\boldsymbol{z}}$. This flow may be described by defining an axisymmetric streamfunction, $\psi(\sigma, \theta)$, as in Batchelor (1967), and writing the velocity in the radial, polar and azimuthal directions respectively as

$$
u=\frac{1}{\sigma^{2} \sin \theta} \frac{\partial \psi}{\partial \theta}, \quad v=-\frac{1}{\sigma \sin \theta} \frac{\partial \psi}{\partial \sigma}, \quad w=-\frac{1}{\operatorname{Ro}}\left(\frac{\psi}{\sigma \sin \theta}+\frac{\sigma \sin \theta}{2}\right) .
$$

(The second term in brackets in the expression for $w$ is included here as we are working in the non-inertial frame of reference of the rotating fluid.) The incompressibility condition $(2.1 a)$ is automatically satisfied. Taylor (1922) proceeded by posing that the streamfunction be of the separable form $\psi=f(\sigma) \sin ^{2} \theta$. Substitution into the curl of $(2.1 b)$, thus removing the pressure gradient, leads to either the trivial solution $f=0$ or that $f$ must satisfy

$$
\sigma^{3} f^{\prime \prime \prime}-2 \sigma^{2} f^{\prime \prime}-2 \sigma f^{\prime}+8 f+\frac{\sigma^{2}}{\mathrm{Ro}^{2}}\left(\sigma f^{\prime}-2 f\right)=0
$$

where a dash denotes differentiation with respect to $\sigma$. In the frame of reference rotating with the fluid, but translating with a sphere of radius $\delta$ that is not precessing in the laboratory frame, the no-normal flow and no-precession boundary conditions on the boundary of the sphere, together with the far-field velocity boundary condition, are

$$
u(\delta, \theta)=0, \quad w(\delta, \theta)=-\frac{\delta \sin \theta}{2 \mathrm{Ro}}, \quad \lim _{\sigma \rightarrow \infty} \boldsymbol{u}(\sigma, \theta)=(-\cos \theta, \sin \theta, 0) .
$$

Equivalently, in terms of $f$

$$
f(\delta)=0, \quad \lim _{\sigma \rightarrow \infty} \frac{f(\sigma)}{\sigma^{2}}=-\frac{1}{2} .
$$

The pressure field is given by

$$
p(\sigma, \theta)=\left(2 f f^{\prime \prime}-f^{\prime 2}+\frac{f^{2}}{\mathrm{Ro}^{2}}\right) \frac{\sin ^{2} \theta}{2 \sigma^{2}}-\frac{2 f^{2}}{\sigma^{4}}+\frac{1}{2},
$$

where the pressure $p \rightarrow\left(8 \mathrm{Ro}^{2}\right)^{-1} \sigma^{2} \sin ^{2} \theta$, the hydrostatic pressure field, as $\sigma \rightarrow \infty$. The vorticity field, $\boldsymbol{\omega} \equiv \nabla \times \boldsymbol{u}$, is

$$
\boldsymbol{\omega}=-\frac{1}{\mathrm{Ro}}\left[\cos \theta\left(\frac{2 f}{\sigma^{2}}+1\right) \hat{\boldsymbol{\sigma}}-\sin \theta\left(\frac{f^{\prime}}{\sigma}+1\right) \hat{\boldsymbol{\theta}}\right]-\frac{\sin \theta}{\sigma}\left(f^{\prime \prime}-\frac{2 f}{\sigma^{2}}\right) \hat{\boldsymbol{\phi}} .
$$



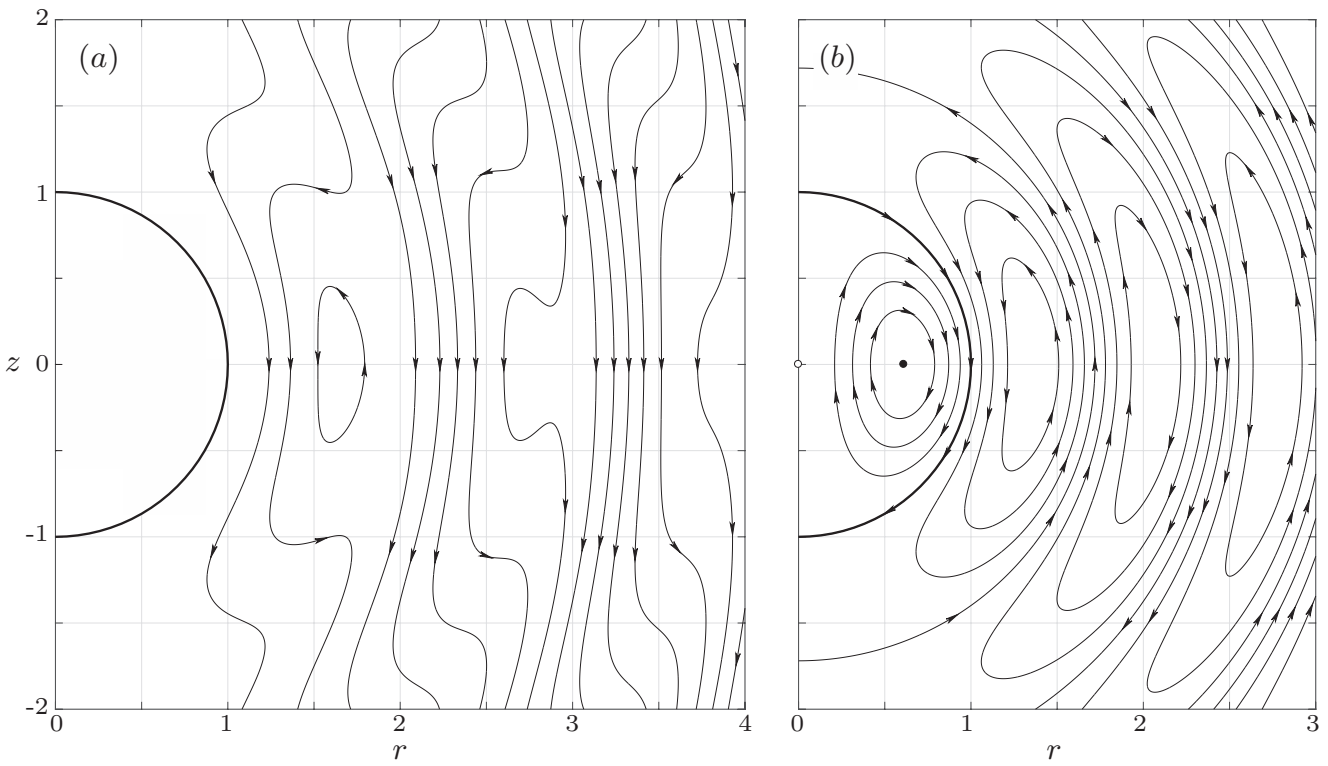

FIgURE 2: (a) Streamlines around a unit sphere translating steadily along the axis of rotation $(r=0)$ of a rotating fluid in the reference frame of the sphere. Closed streamlines show that fluid is being transported with the sphere. (b) Streamlines around a sphere of vanishing size (white circle at the origin) translating steadily along this axis of rotation of a rotating fluid in the frame of reference of the main body of fluid. Taylor (1922) observed that the streamlines near the origin resemble those of a Hill's spherical vortex (Hill 1894) but described the analogy between the flow and a spherical vortex as 'only superficial'.

The solution to (2.3) that satisfies $(2.4 b)$ may be written as

$$
\begin{aligned}
f(\sigma)=-\frac{\sigma^{2}}{2}+\frac{1}{2 \sigma}\left\{\left[\delta^{3}+c(\sigma-\delta)\right] \cos \right. & \left(\frac{\sigma-\delta}{\mathrm{Ro}}\right) \\
& \left.+\frac{\left[\delta^{3} \sigma-c\left(\delta \sigma+\mathrm{Ro}^{2}\right)\right]}{\mathrm{Ro}} \sin \left(\frac{\sigma-\delta}{\mathrm{Ro}}\right)\right\},
\end{aligned}
$$

for an arbitrary constant $c$. The remaining no-slip boundary condition in the polar direction, $v(\delta, \theta)=0$, is satisifed when $f^{\prime}(\delta)=0$ forcing $c=\delta^{2}-3 \mathrm{Ro}^{2}$, and this together with (2.7) can be shown to be equal to Taylor's solution. The no-precession condition and the no-slip boundary condition in the polar direction are physically motivated choices to close the system. The conditions are no-slip conditions applied to an inviscid fluid in the expectation that the inviscid solution will closely approximate the full viscous solution where no-slip conditions would be rigorously valid.

The streamlines for $\delta=1, \operatorname{Ro}=(2 \pi)^{-1}$ are shown in figure $2 a$. The nonlinear wavefield in the fluid can be observed (cf. figure 2 Taylor 1922) as can the closed streamlines that show that fluid is carried with the sphere. Taylor observed that it is not clear how such a flow may be set up; indeed the radiation condition is not everywhere satisfied throughout the wavefield which prohibits the wavefield being created by the towing of the sphere alone. This observation is consistent with the later findings of Stewartson (1958) and Lighthill (1967) who both considered the wavefields built up by motion due to localized 
forcing along the axis of a rotating fluid into initially quiescent fluid and concluded that only columnar modes could exist ahead of the forcing.

Taylor (1922) observed that his analytical solution still exhibited waves even in the limit that the sphere has a vanishingly small radius, i.e., in the limit $\delta \rightarrow 0$. Figure $2 b$ shows this solution, in the laboratory frame of reference. The sphere is instantaneously located at the origin, indicated by a white circle. Taylor (1922) observed that the streamlines near the origin resemble those of a Hill's spherical vortex (Hill 1894). Here the rotation rate has been chosen such that this apparent spherical vortex has a unit radius (given by the largest $R_{0}$ that satisfies $\operatorname{Ro} \sin \left(\mathrm{Ro}^{-1}\right)=\cos \left(\mathrm{Ro}^{-1}\right)$, i.e., $\left.\mathrm{Ro} \approx 0.223\right)$. The boundary of the apparent spherical vortex is shown in bold. Taylor (1922) describes this analogy with Hill's spherical vortex as superficial as the vortex can only exist when the flow is rotating and a vanishingly small sphere is translating steadily, without precession, along the axis of rotation.

\subsection{Hill's spherical vortex in a rotating fluid}

The classical Hill's spherical vortex is a spherical region of rotational fluid that propagates through an irrotational ambient fluid. The flow is found by constructing solutions inside and outside the spherical vortex and enforcing pressure and velocity continuity across the boundary of the two regions. As with the no-slip conditions enforced on the solid sphere in Taylor's solution, continuity of velocity is enforced across the boundary of the two inviscid solutions, even though this is not a strict requirement, in the expectation that the solution will closely approximate the behaviour of a real viscous fluid. In a frame of reference moving with the spherical vortex, Hill's solution is given by

$$
\begin{gathered}
\boldsymbol{u}= \begin{cases}\frac{3 \cos \theta}{2}\left(1-\sigma^{2}\right) \hat{\boldsymbol{\sigma}}-\frac{3 \sin \theta}{2}\left(1-2 \sigma^{2}\right) \hat{\boldsymbol{\theta}} & \sigma \leqslant 1 \\
-\frac{\cos \theta}{\sigma^{3}}\left(\sigma^{3}-1\right) \hat{\boldsymbol{\sigma}}+\frac{\sin \theta}{2 \sigma^{3}}\left(2 \sigma^{3}+1\right) \hat{\boldsymbol{\theta}} & \sigma>1 .\end{cases} \\
p= \begin{cases}-\frac{9 \sigma^{2}\left(3-2 \sigma^{2}\right)}{8} \sin ^{2} \theta+\frac{9 \sigma^{2}\left(2-\sigma^{2}\right)}{8}-\frac{5}{8} & \sigma \leqslant 1 \\
-\frac{3\left(4 \sigma^{3}-1\right)}{8 \sigma^{6}} \sin ^{2} \theta+\frac{2 \sigma^{3}-1}{2 \sigma^{6}} & \sigma>1 .\end{cases}
\end{gathered}
$$

where the arbitrary pressure constant has been chosen without loss of generality such that $p \rightarrow 0$ as $\sigma \rightarrow \infty$. The solution is in the frame of reference of the spherical vortex and so the velocity in the far-field tends to $-\hat{\boldsymbol{z}}$. The solution is axisymmetric and swirlfree and the velocity can be represented by a streamfunction, $\psi$, of the form of $(2.2 a-b)$, where

$$
\psi= \begin{cases}\frac{3 \sigma^{2}\left(1-\sigma^{2}\right)}{4} \sin ^{2} \theta & \sigma \leqslant 1 \\ -\frac{\left(\sigma^{3}-1\right)}{2 \sigma} \sin ^{2} \theta & \sigma>1\end{cases}
$$

and $w=0$. The arbitrary constant that may be added to the streamfunction is chosen such that $\psi=0$ on the boundary of the spherical vortex. The pressure and velocity fields are continuous across the boundary of the spherical vortex, $\sigma=1$.

We now make the following observation; if $\boldsymbol{u}(\sigma, \theta)$ together with a corresponding pressure field, $p(\sigma, \theta)$, solves the non-rotating Euler equations and $\boldsymbol{u}$ can be represented by a streamfunction $\psi(\sigma, \theta)$ in the form of $(2.2 a-b)$ with $w=0$, then

$$
\boldsymbol{u}=\left(\frac{1}{\sigma^{2} \sin \theta} \frac{\partial \psi}{\partial \theta},-\frac{1}{\sigma \sin \theta} \frac{\partial \psi}{\partial \sigma},-\frac{\sigma \sin \theta}{2 \mathrm{Ro}}\right)
$$


solves the rotating Euler equations with the same pressure field $p$. This is because the swirling component in $(2.10), w=-(2 \mathrm{Ro})^{-1} \sigma \sin \theta$, exactly cancels the background rotation of the fluid. Thus, we have that $(2.10)$ with $\psi$ given by Hill's inner non-rotating solution $((2.9)$ for $\sigma \leqslant 1)$, and the pressure, $p((2.8 b)$ for $\sigma \leqslant 1)$ automatically satisfies the incompressibility condition and the rotating equations of motion $(2.1 a-b)$. The solution is Hill's non-rotating spherical vortex described in a rotating frame of reference. To distinguish this solution from the classical solution we refer to it as the 'swirling' Hill's spherical vortex, even though the azimuthal component of the velocity field exactly cancels the background rotation of the fluid. The observation that Taylor's sphere in his experiments did not precess about the axis of rotation gives rise to the possibility that there may exist a form of Taylor's solution that can be matched onto the swirling Hill's spherical vortex by enforcing a different choice of polar velocity boundary condition to Taylor's no-slip condition when setting $c$ in (2.7).

To match a solution of the form (2.2) onto the swirling Hill's spherical vortex such that the velocity field is continuous across the boundary $\sigma=1$, we require a solution to $(2.3)$ that satisfies, for $\delta=1$, the conditions $(2.4 b)$ and yields $v(1, \theta)=\frac{3}{2} \sin \theta$. We therefore require $f^{\prime}(1)=-\frac{3}{2}$ and hence the solution is given by $(2.7)$ with $\delta=1, c=1$, so that

$$
f(\sigma)=-\frac{\sigma^{2}}{2}+\frac{1}{2 \sigma}\left\{\sigma \cos \left(\frac{\sigma-1}{\mathrm{Ro}}\right)-\operatorname{Rosin}\left(\frac{\sigma-1}{\mathrm{Ro}}\right)\right\} .
$$

Substitution of this solution at $\sigma=1$ into (2.5) shows that the pressure on the boundary is given by $p(1, \theta)=\frac{1}{2}-\frac{9}{8} \sin ^{2} \theta$, exactly matching the pressure on the boundary of the swirling Hill's spherical vortex (see $(2.8 b)$ at $\sigma=1$ ). The solution has the property that Hill's classical solution is recovered in the limit Ro $\rightarrow \infty$. Thus, we have a complete steady solution to the nonlinear rotating Euler equations whose inner solution is Hill's spherical vortex with an additional swirling component in the azimuthal direction that cancels out the background rotation of the fluid. This inner solution matches onto an outer solution, with continuous velocity and pressure across the boundary of the vortex. In the far-field the velocity tends to the free-stream velocity $-\hat{\boldsymbol{z}}$ and pressure tends to the hydrostatic pressure field $p=\left(8 \mathrm{Ro}^{2}\right)^{-1} \sigma^{2} \sin ^{2} \theta$. We observe that $\psi$ is even in the Rossby number and so the waves that form in a meridional plane ( $\phi=$ const.) oscillate according only to the magnitude of the rotation of the system, and not the sign of the direction of rotation, as might be expected on physical grounds. Similarly, as a result of $(2.2 c)$ and (2.11), the azimuthal velocity is odd in the Rossby number and so the azimuthal flow field is reversed if the sign of the direction of rotation of the system is reversed. We see from (2.11) that the wavelength of the inertial waves in the outer fluid is $2 \pi$ Ro as in Taylor (1922).

Figure $3(a)-(c)$ shows streamlines in the $(r, z)$ meridional plane of Hill's spherical vortex and the flow outside the spherical vortex for three different values of the Rossby number. The streamlines represent the flow relative to the translating spherical vortex. The meridional velocity components are always zero at $r=2^{-1 / 2}, z=0$, indicated by black circles. Plot $(a)$ is of the non-rotating Hill's spherical vortex that corresponds to the limit Ro $\rightarrow \infty$. As the rate of rotation is increased, and the Rossby number reduces from $\operatorname{Ro}=\frac{1}{4}$ in plot $(b)$ to $\operatorname{Ro}=\frac{1}{10}$ in plot $(c)$, inertial waves can be observed in the outer fluid. Below a critical Rossby number it can be seen that these waves begin to overturn. We also observe that closed streamlines appear in the outer fluid in plot $(c)$. These closed streamlines show that above a critical rotation rate, regions of fluid are transported with the spherical vortex, externally to the spherical vortex in the form of concentric vortex rings. 

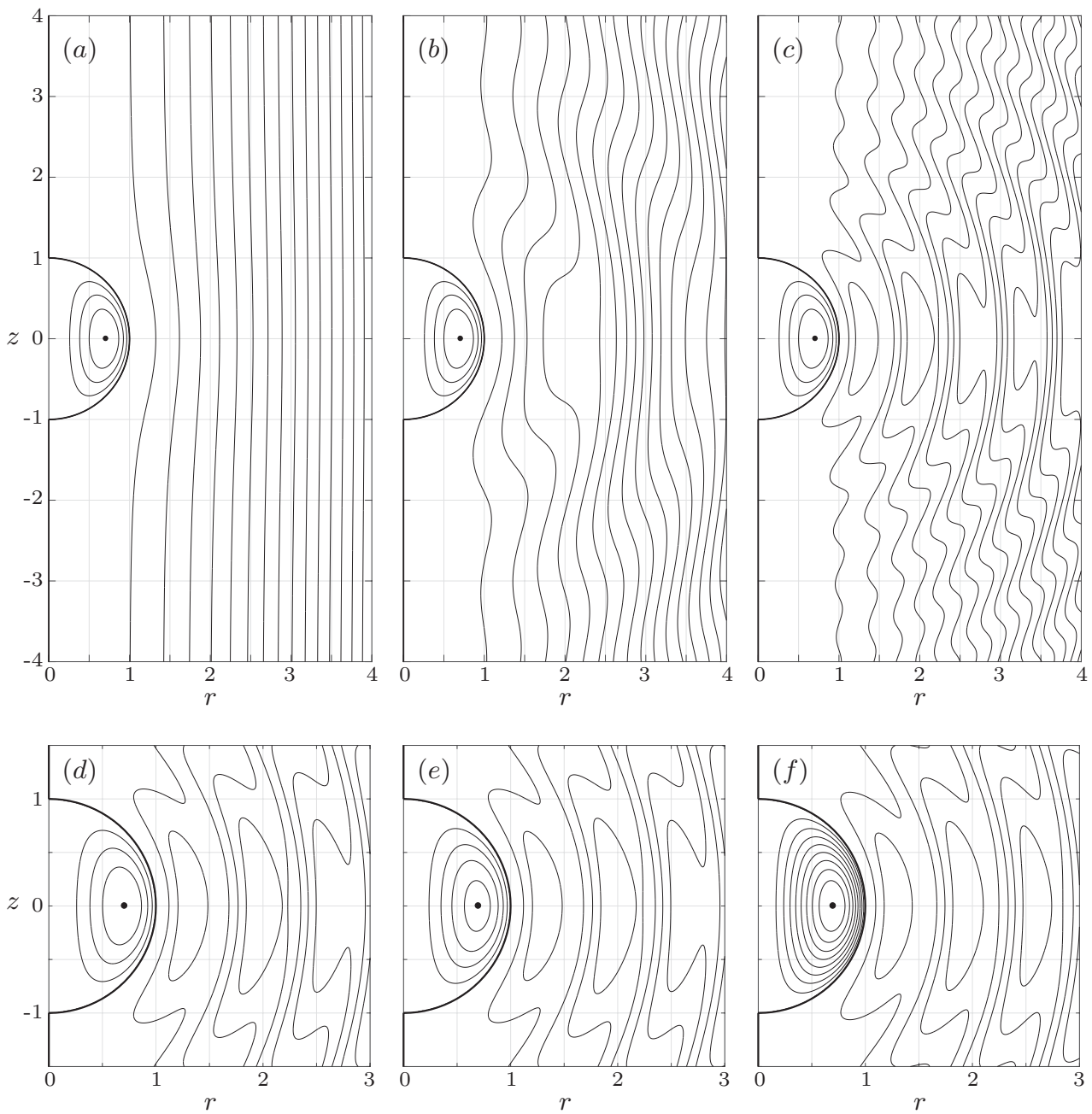

Figure 3: $(a)-(c)$ : Plots of the streamfunction for Hill's spherical vortex for Ro $=\infty$, $\frac{1}{4}, \frac{1}{10}$ for $(a)-(c)$ respectively. The meridional stagnation point in the spherical vortex is indicated by a small black circle and is located at $r=2^{-1 / 2}, z=0$. In plot $(c)$ the formation of closed streamlines externally to the spherical vortex may be observed, indicating regions of fluid that are transported with the spherical vortex. $(d)-(f)$ : Plots of the streamfunction for swirling spherical vortices for Ro $=\frac{1}{10}$. The parameters shown are: $(d) \alpha=0, \lambda=\frac{15}{2}$ (Hill's spherical vortex); (e) $\alpha=\frac{\pi}{2}, \lambda=\frac{15}{2} ;(f) \alpha=\frac{\pi}{2}, \lambda=15$.

\subsection{Swirling spherical vortices in a rotating fluid}

We now generalize the method of $\S 2.2$ for the family of swirling spherical vortices identified by Moffatt (1969). The spherical vortex, for $\sigma \leqslant 1$, is described in terms of a streamfunction by

$$
u=\frac{1}{\sigma^{2} \sin \theta} \frac{\partial \psi}{\partial \theta}, \quad v=-\frac{1}{\sigma \sin \theta} \frac{\partial \psi}{\partial \sigma}, \quad w=-\frac{\alpha \psi}{\sigma \sin \theta}-\frac{\sigma \sin \theta}{2 \mathrm{Ro}} .
$$

As with Hill's solution, the streamfunction has the same separable form as Taylor's streamfunction, specifically $\psi=F(\sigma) \sin ^{2} \theta$, where for $(\lambda, \alpha) \in \mathbb{R}^{2}$ we have the doubly- 
infinite family of spherical vortices given by

$$
F(\sigma)=\frac{\lambda}{\alpha^{2}}\left[\frac{\sigma \alpha \cos (\sigma \alpha)-\sin (\sigma \alpha)}{\sigma(\alpha \cos \alpha-\sin \alpha)}-\sigma^{2}\right] .
$$

The corresponding pressure field is given by (2.5) with $f$ replaced by $F$ and Ro replaced by $\alpha^{-1}$. It follows from (2.12) that $\alpha$ is a measure of the degree of swirl in the spherical vortex. The special case of Hill's spherical vortex ring is recovered in the limit $\alpha \rightarrow 0$, $\lambda=\frac{15}{2}$. No-normal flow and no-precession conditions are satisfied on the boundary of the swirling spherical vortices as $F(1)=0$, and hence $u(1, \theta)=0$ and $w(1, \theta)=$ $-(2 \mathrm{Ro})^{-1} \sin \theta$. The conditions of continuity of pressure and polar velocity across the boundary are satisfied if a solution for $f$, given by (2.7) with $\delta=1$, can be found such that $f^{\prime}(1)=F^{\prime}(1)$ and this condition is satisfied when

$$
c=1+2 \operatorname{Ro}^{2}\left[\frac{\lambda}{\alpha^{2}}\left(3+\frac{\alpha^{2} \sin \alpha}{\alpha \cos \alpha-\sin \alpha}\right)-\frac{3}{2}\right] .
$$

(Note that the result of $\S 2.2, c=1$, is recovered in the limit $\alpha \rightarrow 0, \lambda=\frac{15}{2}$.) Hence, any swirling spherical vortex given by (2.12) and (2.13) may be matched onto an oncoming stream in a rotating fluid whose far-field velocity tends to $-\hat{\boldsymbol{z}}$ with continuous pressure and velocity across the boundary of the spherical vortex at $\sigma=1$. We also see that the outer flow is a singly-infinite family given by (2.14) and is equal to the outer flow of the swirling Hill's spherical vortex solution for all solutions with $c=1$, i.e., for all $(\lambda, \alpha) \in \mathbb{R}^{2}$ such that the quantity in square brackets in (2.14) is zero.

Figure $3(d)-(f)$ shows streamlines in the $(r, z)$ meridional plane of the swirling spherical vortices for Ro $=\frac{1}{10}$ and: $(d) \alpha=0, \lambda=\frac{15}{2}$ Hill's swirling spherical vortex; $(e) \alpha=\frac{\pi}{2}$, $\lambda=\frac{15}{2} ;(f) \alpha=\frac{\pi}{2}, \lambda=15$. It can be seen that the stronger of the two swirling Moffatt solutions $(f)$ corresponds to a larger amplitude wavefield in the outer fluid, with three closed streamlines in the image shown.

\subsection{Sibling vortices}

The critical rotation rate at which the onset of overturning is observed may be found by considering the turning points of the streamlines. If a given streamline, $\psi=$ const. for $\sigma>1$, is parameterized by $\theta=\theta(\sigma)$ then a necessary condition for overturning is $\mathrm{d} \theta(\sigma) / \mathrm{d} \sigma=0$, that is, when

$$
\begin{aligned}
2 \operatorname{Ro}^{2} \sigma^{3}+\left\{\operatorname{Ro}^{2} \sigma\right. & \left.+(c-1)\left[\sigma^{2}+\operatorname{Ro}^{2}(\sigma-1)\right]\right\} \cos \left(\frac{\sigma-1}{\operatorname{Ro}}\right) \\
& +\left\{\sigma^{2}-\operatorname{Ro}^{2}-(c-1)\left[\operatorname{Ro}^{2}-\sigma(\sigma-1)\right]\right\} \sin \left(\frac{\sigma-1}{\operatorname{Ro}}\right)=0 .
\end{aligned}
$$

This expression has a different number of branches of solution for a given Rossby number, Ro, as is shown in figure $4 a$ for $c=1$. There is therefore in this case a minimum rate of rotation below which no closed streamlines in the outer fluid are formed and no fluid is carried along with the spherical vortex. The first critical rotation rate occurs when $\mathrm{d} \operatorname{Ro}(\sigma) / \mathrm{d} \sigma=0$ where $\operatorname{Ro}=\operatorname{Ro}(\sigma)$ is determined by $(2.15 a)$. This condition is given by

$$
\begin{aligned}
6 \operatorname{Ro}^{3} \sigma+\operatorname{Ro}[\sigma+(c-1)(\sigma+1)] & \cos \left(\frac{\sigma-1}{\operatorname{Ro}}\right) \\
+ & {\left[\operatorname{Ro}^{2}+(c-1)\left(\operatorname{Ro}^{2}-\sigma\right)\right] \sin \left(\frac{\sigma-1}{\operatorname{Ro}}\right)=0 . }
\end{aligned}
$$



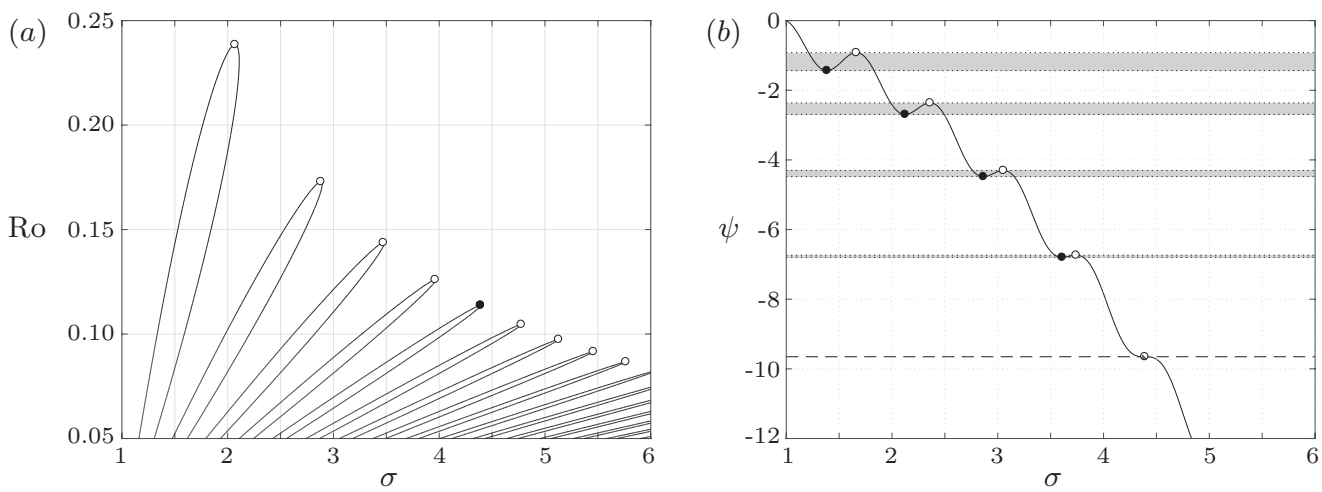

Figure 4: $(a)$ Zeros of (2.15a) for $c=1$; as the rotation rate increases the Rossby number, Ro, decreases and new branches of the solution are found. The critical rotation rates at which new branches of solution are found are given by zeros of the system (2.15), indicated by circles. The fifth critical Rossby $\mathrm{Ro}=\mathrm{Ro}_{c}^{(5)} \approx 0.114$ is indicated by the black circle. (b) The streamfunction for $\theta=\pi / 2$ and $\operatorname{Ro}=\operatorname{Ro}_{c}^{(5)}$. This plot corresponds to the $z=0$ transect of figure 5 . The black circles that occur at local minima in $\psi$ correspond to black stagnation points in figure 5 . The white circles that occur at local maxima in $\psi$ correspond to the white stagnation points in figure 5. For $\psi$ lying between pairs of black and white turning points, indicated by the grey regions, the function $\sigma(\psi)$ is multi-valued and this corresponds to closed streamlines in the flow. At the chosen Rossby number the fifth set of closed streamlines is about to appear at the inflection point indicated by the dashed line, corresponding to the cusp at $r \approx 4.39$ (black-white circle) in figure 5 .

The critical points determined by simultaneous solutions of $(2.15 a)$ and $(2.15 b)$ are shown for $c=1$ as circles in figure $4 a$ and we denote the critical Rossby numbers as Ro $=$ $\operatorname{Ro}_{c}^{(n)}$ and the corresponding critical radii as $\sigma=\sigma_{c}^{(n)}$ for $n=1,2,3, \ldots$. The first critical rotation rate, $\mathrm{Ro}_{c}^{(1)}$, that represents the minimum rotation rate for which a closed streamline forms in the flow is found numerically, for Hill's spherical vortex, to be $\operatorname{Ro}_{c}^{(1)} \approx$ 0.239 . This rotation rate lies between those shown in figure $3 b$ and $3 c$. The corresponding radius, $\sigma_{c}^{(1)} \approx 2.07$ and $\theta=\pi / 2$ gives the location at which the overturning first occurs. For a given Rossby number, the associated number of branches of solutions of $(2.15 \mathrm{a})$ corresponds to the number of closed streamlines in the flow outside the spherical vortex, and hence corresponds to the number of regions of fluid that are advected with the spherical vortex.

The value of the streamfunction on the closed streamlines and the location of stagnation points in the flow can be found by considering $\psi$ on $\theta=\pi / 2$. Figure $4 b$ shows $\psi(\sigma, \pi / 2)$ for $\sigma>1$ and $\mathrm{Ro}=\mathrm{Ro}_{c}^{(5)} \approx 0.114$, the fifth critical rotation rate (indicated by the black circle in figure $4 a$ ). The turning points in $\psi$ can be seen to appear in pairs of local minima (black circles) and local maxima (white circles). For values of $\psi$ between the local minima and maxima, indicated by the grey bands, the function $\sigma(\psi)$ is multi-valued and this corresponds to closed streamlines in the flow. As the flow considered is exactly at the fifth critical rotation rate, the fifth pair of local minima and maxima coincide at the inflection in $\psi$ where $\sigma \approx 4.39$ and $\psi \approx-9.65$.

Figure 5 shows streamlines of the flow for a swirling Hill's spherical vortex in a rotating fluid at $\mathrm{Ro}=\mathrm{Ro}_{c}^{(5)}$ corresponding to the rotation rate in figure $4 b$. The stagnation point in the spherical vortex is, as in the classical solution, at $\sigma=2^{-1 / 2}, \theta=\pi / 2$. In the outer 


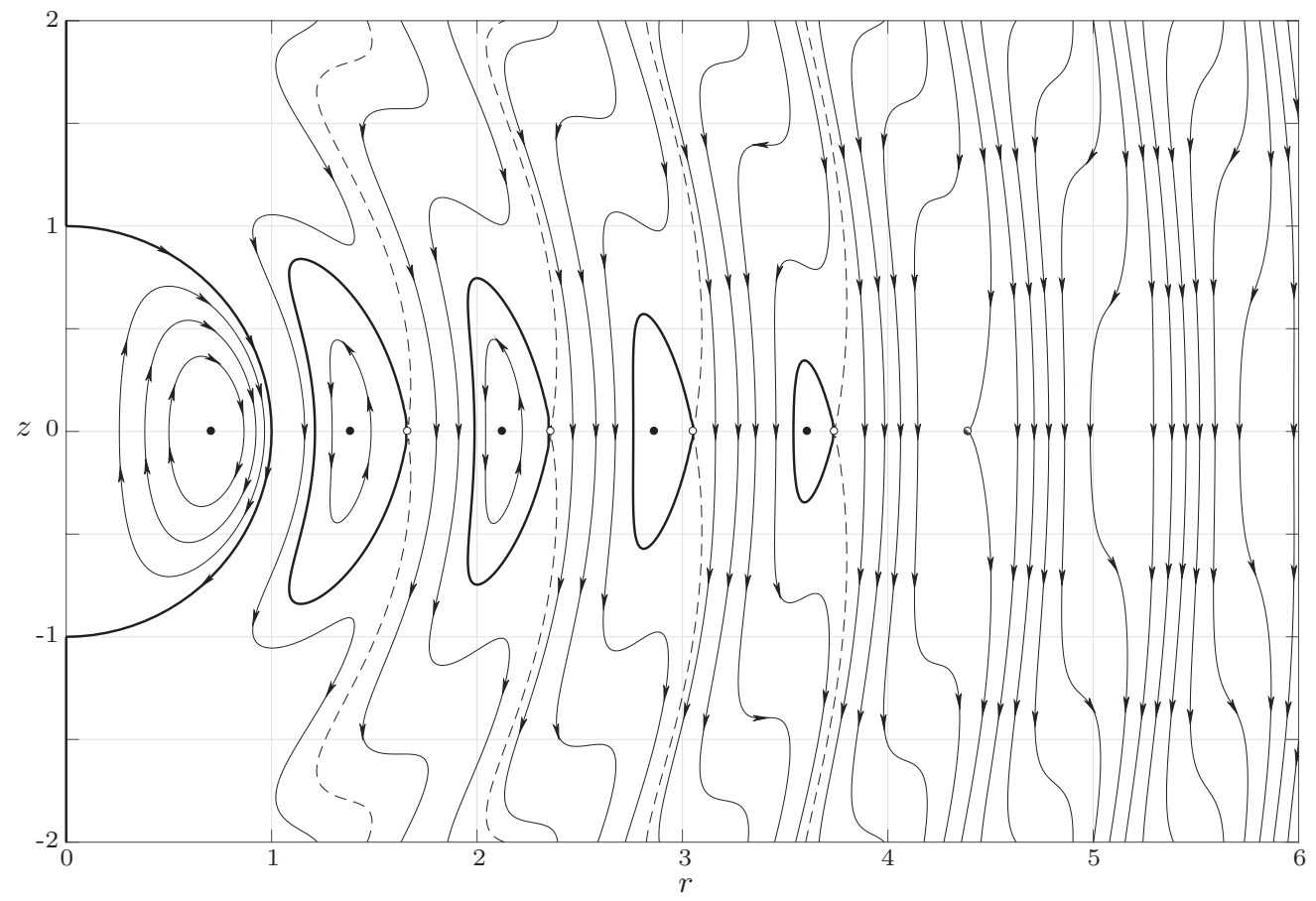

FIGURE 5: A meridional slice through the flow field in the frame of reference moving with a swirling Hill's spherical vortex ring for $\mathrm{Ro}=\mathrm{Ro}_{c}^{(5)} \approx 0.114$. This is the fifth critical Rossby number, we can see four sibling vortex rings (bold lines) have been created in the outer fluid and there is a cusp that has formed (black-white circle at $r \approx 4.39, z=0$ ) on a streamline that will form the next sibling vortex ring when $\mathrm{Ro}<\mathrm{Ro}_{c}^{(5)}$.

flow there can be seen to be four closed streamlines, indicated in bold. We observe that the direction of advection around these closed streamlines (anti-clockwise) is opposite to that in the spherical vortex (clockwise), though the vorticity in the closed streamlines may change sign (see (2.6)). The black stagnation points in the closed streamlines correspond to the black local minima in figure $4 b$. The white stagnation points, on the boundary of the closed streamlines, correspond to the local maxima in $4 b$. A fifth 'closed streamline' is about to form at the cusp indicated by the black-white circle at $\sigma \approx 4.39$. This corresponds to the inflection in $\psi$ in figure $4 b$.

\section{Conclusions}

Following Taylor's (1922) observation that his analytical solution for streamlines around a steadily translating, vanishingly small, sphere on the axis of rotation of a rotating fluid resemble those of a Hill's spherical vortex (Hill 1894), we have derived explicit solutions of the rotating Euler equations that support steadily propagating spherical vortices. The inner solution comprises spherical vortices that are members of a doubly-infinite family of solutions whose boundary swirls in such a way as to exactly cancel the background rotation of the fluid, in that way mimicking the behaviour of the sphere towed through the rotating tank in Taylor's experiments (see also experimental observations in developed vortex rings in rotating fluids (e.g., Eisenga 1997; Verzicco et al. 1996)). This inner solution matches onto an outer free-stream solution that exhibits nonlinear inertial waves. 
As the rotation rate is increased the amplitude of the waves is observed to grow until, at a critical rotation rate wave-overturning may be observed and closed streamlines form in the outer fluid. The closed streamlines represent vortex rings that are concentric to the spherical vortex on the axis and which propagate with the spherical vortex. As the rotation rate is increased beyond subsequent critical Rossby numbers, new 'sibling' vortex rings are added to the vortex ring family propagating along the axis of rotation.

The authors would like to thank an anonymous referee for drawing our attention to the solutions of Moffatt (1969).

\section{REFERENCES}

Batchelor, G. K. 1967 An introduction to fluid dynamics. Cambridge University Press.

EISEnGA, A. H. M. 1997 Dynamics of a vortex ring in a rotating fluid. PhD thesis, Eindhoven University of Technology.

FrAenkel, L. E. 1970 On steady vortex rings of small cross-section in an ideal fluid. Proc. Roy. Soc. A 316, 29-62.

FrAenkel, L. E. 1972 Examples of steady vortex rings of small cross-section in an ideal fluid. J. Fluid Mech. 51, 119-135.

Hill, M. J. M. 1894 On a spherical vortex. Phil. Trans. Roy. Soc. A 185, 213-245.

Lighthill, M. J. 1967 On waves generated in dispersive systems by travelling forcing effects, with applications to the dynamics of rotating fluids. J. Fluid Mech. 27(4), 725-752.

Moffatt, H. K. 1969 The degree of knottedness of tangled vortex lines. J. Fluid Mech. 35(1), $117-129$.

Moffatt, H. K. \& Moore, D. W. 1978 The response of Hill's spherical vortex to a small axisymmetric disturbance. J. Fluid Mech. 87(4), 749-760.

Norbury, J. 1973 A family of steady vortex rings. J. Fluid Mech. 57(3), 417-431.

Pozrikidis, C. 1986 The nonlinear instability of Hill's vortex. J. Fluid Mech. 168, 337-367.

Protas, B. \& ElCRAT, A. 2016 Linear stability of Hill's vortex to axisymmetric perturbations. J. Fluid Mech. 799, 579-602.

Stewartson, K. 1958 On the motion of a sphere along the axis of a rotating fluid Q. J. Mech. Appl. Math. 11(1), 39-51.

TAYlor, G. I. 1922 The motion of a sphere in a rotating liquid. Proc. Roy. Soc. A 102(715), 180-189. 1922.

Verzicco, R., Orlandi, P., Eisenga, A. H. M., van Heijst, G. J. F. \& Carnevale, G. F. 1996 Dynamics of a vortex ring in a rotating fluid. J. Fluid Mech. 317, 215-239. 